\title{
Genetics of Menstrual Migraine: The Epidemiological Evidence
}

\author{
Michael Bjørn Russell
}

Published online: 10 August 2010

(C) The Author(s) 2010. This article is published with open access at Springerlink.com

\begin{abstract}
Approximately one of every three to five women with migraine without aura experience migraine attacks in relation to menstruation. The International Classification of Headache Disorders, 2nd Edition provides appendix diagnoses for pure and menstrually related migraine without aura that need further validation. Probands with menstrual migraine might have more affected relatives than probands with nonmenstrual migraine. However, precise epidemiological, family, and twin data still are lacking.
\end{abstract}

Keywords Menstrual migraine $\cdot$ Epidemiology $\cdot$ Genetics

\section{Introduction}

The definition of menstrual migraine varied before the introduction of the International Headache Society's (IHS) Classification and Diagnostic Criteria for Headache Disorders and Facial Pain in 1988 [1]. The IHS classification did not classify menstrual migraine as a specific type of headache, but had a comment in relation to migraine without aura: "Migraine without aura may occur almost exclusively at a particular time of the menstrual cycle-so-called menstrual migraine. Generally accepted criteria for this entity are not

M. B. Russell

Head and Neck Research Group, Research Centre,

Akershus University Hospital,

Lørenskog,

Oslo 1478, Norway

M. B. Russell ( $\square)$

Faculty Division, Akershus University Hospital,

University of Oslo,

Nordbyhagen,

Oslo 1474, Norway

e-mail: m.b.russell@medisin.uio.no available. It seems reasonable to demand that $90 \%$ of attacks should occur between 2 days before menses and the last day of menses, but further epidemiological knowledge is needed" [1]. The revision of the IHS classification, the International Classification of Headache Disorders, 2nd Edition (ICDH-II) from 2004, does not include menstrual migraine within the main body of the classification [2]. However, it provides diagnostic criteria for pure menstrual migraine without aura and menstrually-related migraine without aura in the appendix. Pure menstrual migraine without aura is defined as migraine without aura that occurs exclusively on day 1 of menstruation \pm 2 days in at least two out of three menstrual cycles. Menstrually related migraine without aura additionally has attacks of migraine without aura in the nonmenstrual period. Further scientific evidence is needed before these terms can be part of future headache classifications.

\section{Relation to Female Hormones}

Some elegant studies from the early 1970 s showed that changes in estrogen levels could precipitate an attack of migraine [3-6]. A population-based survey on migraine with physician-conducted interviews found that menstrual migraine occurred especially in females with similar age at onset of migraine without aura and menarche, while this association was not present in migraine with aura. Attacks in relation to ovulation could not be demonstrated in migraine either with or without aura [7]. This may be because the peak of estrogen at ovulation is of short duration and the fall of serum estrogen must be preceded by several days of exposure to high levels of estrogens to precipitate the attack [5]. Both migraine without aura and migraine with aura improve during pregnancy [7-11]. The results suggest that female hormones are important as a 
precipitating factor in migraine without aura, but less so in migraine with aura.

\section{Epidemiology}

The prevalence of migraine before puberty is equal in boys and girls [12]. After menarche and during women's reproductive years, the gender ratio shows a two- to threefold preponderance of women, because the prevalence of migraine without aura increases much more in women than men $[13,14]$. More than $50 \%$ of women with migraine report an association between menstruation and migraine $[15,16]$. Table 1 shows the prevalence of pure menstrual migraine without aura and menstrually related migraine without aura. The prevalence varies due to different diagnostic criteria and methodology. The prevalence of pure menstrual migraine without aura varies between $7 \%$ and $14 \%$ among female migraineurs, while the prevalence of menstrually related migraine without aura varies from $10 \%$ to $71 \%$ among female migraineurs. Approximately one of every three to five female migraineurs has attacks of migraine without aura in relation to menstruation.

\section{Positive Family History}

Transmission of migraine from parents to children was reported as early as the 17 th century [17]. Since then, numerous studies have reported a positive family history of migraine [18]. A positive family history is imprecise because it does not specify number of affected family members, family size, or relation to the proband. The lifetime prevalence of migraine is $16 \%-21 \%$ in the general population $[10,13,14]$. This causes a positive family history simply by chance in over $80 \%$ of probands with six first-degree relatives (parents, siblings, and children), and one or both parents are affected in over $40 \%$ of the families. Thus, a positive family history does not prove the presence of a genetic factor. Furthermore, a positive family history does not include an interview of the relatives by a physician. Migraine assessed by proband report compared to a clinical interview by a physician is not sufficiently precise because the number of affected relatives is highly underestimated and often misclassified [19]. Thus, a clinical interview by a physician is indispensable in family studies of migraine. Only a single study reports a positive family history of menstrual migraine [20]. This study was based on a questionnaire filled in by female students on behalf of themselves and their families. The study showed that students with menstrual migraine significantly more frequently had 2 or more relatives with migraine than other subtypes of migraine. Thus, probands with menstrual migraine may have more affected relatives than those with nonmenstrual migraine, given the abovementioned shortcomings.

\section{Family and Twins Studies of Migraine without Aura}

An increased familial risk can be caused by genetic as well as environmental factors. The risk among spouses can be used to evaluate this relation because probands and spouses in part share a common environment but differ in genetic constitution [21]. Thus, an increased risk among first-degree relatives and no increased risk among spouses favor importance of genetic factors, while no increased risk among first-degree relatives and spouses favors importance of environmental factors. The relative risk of migraine without aura is increased by a factor of 1.86 in first degree relatives of probands with migraine without aura [22]. Studies of twin pairs are the classical method to investigate the relative importance of genetic and environmental factors. Most twin studies have been case reports or small series; larger samples are limited. Unfortunately, most studies have not discriminated between migraine without aura and migraine with aura. A Danish study included 1013 monozygotic and 1667 dizygotic twin pairs of the same gender from a population-based twin register [23]. The pairwise concordance rate was significantly higher among monozygotic than dizygotic twin pairs $(P<0.05)$. However, environmental factors also seem to play an important role because the pairwise concordance rate in monozygotic twin pairs never reached $100 \%$. Thus, family and twin studies suggest that migraine without aura is caused by a combination of genetic and environmental factors. A complex segregation analysis suggested that migraine without aura has multifactorial inheritance [24]. Unfortunately, family and twin studies on menstrual migraine are lacking.

\section{Conclusions}

Migraine without aura is a syndrome. Pure menstrual migraine without aura and menstrually related migraine without aura most likely are subtypes or subsyndromes of migraine without aura. At least a recent study suggests that menstrual versus nonmenstrual attacks of migraine are more intense and of longer duration in people with menstrual migraine [25•]. Future epidemiological surveys on menstrual migraine in the general population should focus on and work out a more precise definition for menstrual migraine. The current ICHD-II provides excellent provisory criteria, but it is important to record other features that can add to an even more precise diagnosis. Similarly, precise family and twin studies of menstrual migraine based on physician interview in a blinded fashion would be important in the future to elucidate whether pure 


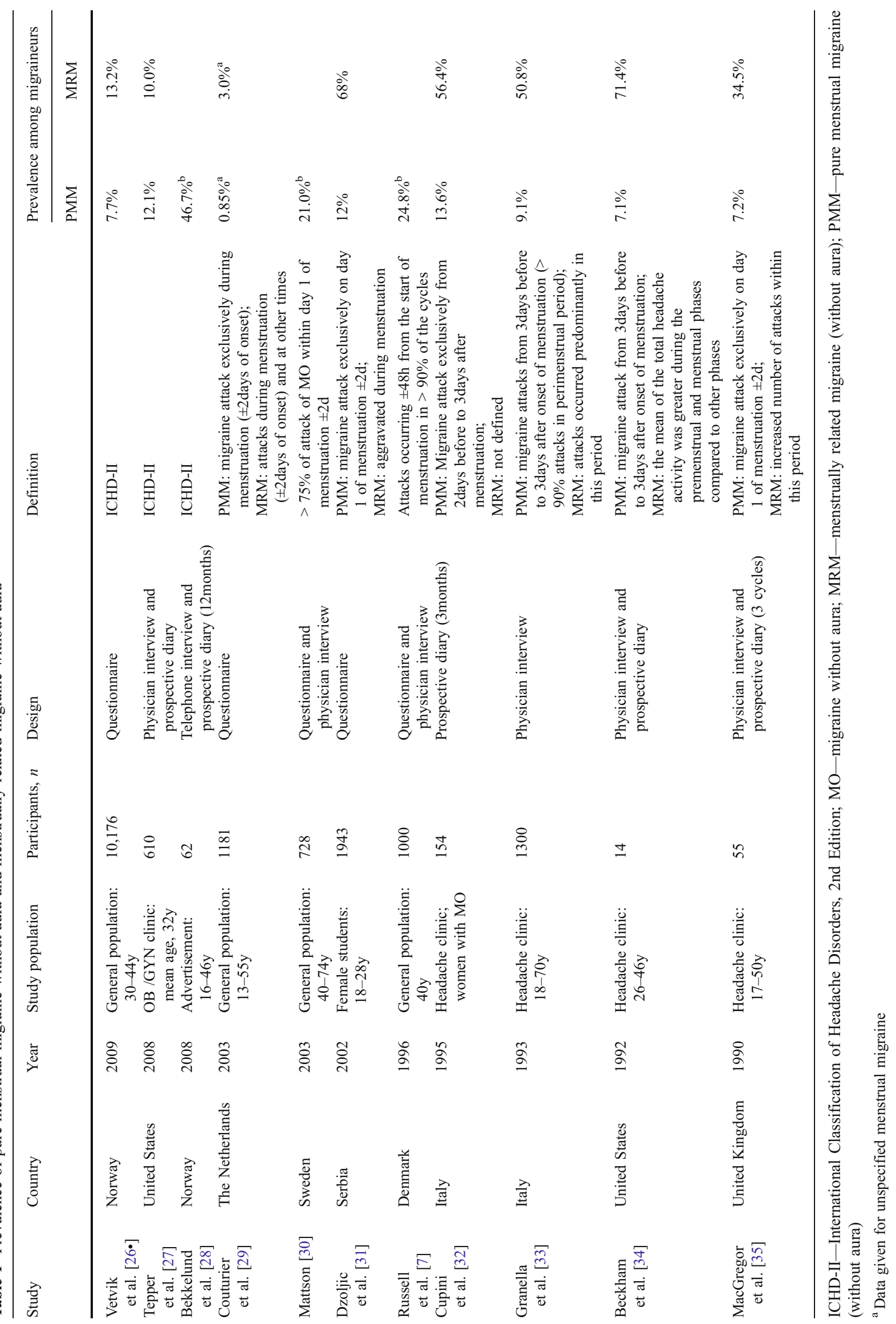


menstrual migraine without aura and menstrually related migraine without aura are specific subforms of migraine without aura or simply variations within the migraine without aura syndrome spectrum.

Disclosure No potential conflict of interest relevant to this article was reported.

Open Access This article is distributed under the terms of the Creative Commons Attribution Noncommercial License which permits any noncommercial use, distribution, and reproduction in any medium, provided the original author(s) and source are credited.

\section{References}

Papers of particular interest, published recently, have been highlighted as:

\section{- Of importance}

1. Classification and diagnostic criteria for headache disorders, cranial neuralgias and facial pain. Headache Classification Committee of the International Headache Society. Cephalalgia 1988, 8(Suppl 7):1-96.

2. Headache Classification Subcommittee of the International Headache Society: The International Classification of Headache Disorders: 2nd edition. Cephalalgia 2004, 24(Suppl 1):9-160.

3. Somerville BW: The role of progesterone in menstrual migraine. Neurology 1971, 21:853-859.

4. Somerville BW: The role of estradiol withdrawal in the etiology of menstrual migraine. Neurology 1972, 22:355-365.

5. Somerville BW: Estrogen-withdrawal migraine. I. Duration of exposure required and attempted prophylaxis by premenstrual estrogen administration. Neurology 1975, 25:239-244.

6. Somerville BW: Estrogen-withdrawal migraine. II. Attempted prophylaxis by continuous estradiol administration. Neurology 1975, 25:245-250.

7. Russell MB, Rasmussen BK, Fenger K, Olesen J: Migraine without aura and migraine with aura are distinct clinical entities: a study of four hundred and eighty-four male and female migraineurs from the general population. Cephalalgia 1996, 16:239-245.

8. Somerville BW: A study of migraine in pregnancy. Neurology 1972, 22:824-828.

9. Silberstein SD, Merriam GR: Estrogens, progestins, and headache. Neurology 1991, 41:786-793.

10. Rasmussen BK, Olesen J: Migraine with aura and migraine without aura: an epidemiological study. Cephalalgia 1992, 12:221-228.

11. Rasmussen BK: Migraine and tension-type headache in the general population: precipitating factors, female hormones, sleep pattern and relation to lifestyle. Pain 1993, 53:65-72.

12. Bille BS: Migraine in school children. A study of the incidence and short-term prognosis, and a clinical, psychological and electroencephalographic comparison between children with migraine and matched controls. Acta Paediatr Suppl 1962, 136:1-151.

13. Rasmussen BK, Jensen R, Schroll M, Olesen J: Epidemiology of headache in a general population-a prevalence study. J Clin Epidemiol 1991, 44:1147-1157.
14. Russell MB, Rasmussen BK, Thorvaldsen P, Olesen J: Prevalence and sex-ratio of the subtypes of migraine. Int J Epidemiol 1995, 24:612-618.

15. Granella F, Sances G, Pucci E, et al.: Migraine with aura and reproductive life events: a case control study. Cephalalgia 2000, 20:701-707.

16. MacGregor EA, Igarashi $\mathrm{H}$, Wilkinson $\mathrm{M}$ : Headaches and hormones: subjective versus objective assessment. Headache Q 1997, 8:126-136.

17. Willis T: Opera Omnia. Amstelaedami: Henricum Wetstenium; 1682.

18. Russell MB: Genetic epidemiology of migraine and cluster headache. Cephalalgia 1997, 17:683-701.

19. Russell MB, Fenger K, Olesen J: The family history of migraine. Direct versus indirect information. Cephalalgia 1996, 16:156-160.

20. Vlajinac HD, Dzoljic ED, Sipetic SB, Kostic VS: Hereditary patterns of Belgrade university female students with migraine and nonmigraine primary headache. J Neurol 2004, 251:973-976.

21. Vogel F, Motulsky AG: Human Genetics: Problems and Approaches, edn 2. Berlin, Heidelberg: Springer-Verlag; 1986.

22. Russell MB, Olesen J: Increased familial risk and evidence of genetic factor in migraine. BMJ 1995, 311:541-544.

23. Gervil M, Ulrich V, Kaprio J, et al.: The relative role of genetic and environmental factors in migraine without aura. Neurology 1999, 53:995-999.

24. Russell MB, Iselius L, Olesen J: Inheritance of migraine investigated by complex segregation analysis. Hum Genet 1995, 96:726-730.

25. - MacGregor EA, Victor TW, Hu X, et al.: Characteristics of menstrual vs nonmenstrual migraine: A post hoc, within-women analysis of the usual-care phase of a nonrandomized menstrual migraine clinical trial. Headache 2010, 50:528-538. This article provides characteristics of menstrual and nonmenstrual migraine in a clinic population.

26. - Vetvik KG, MacGregor EA, Lundqvist C, Russell MB: Selfreported menstrual migraine in the general population. J Headache Pain 2010, 11:87-92. This article provides prevalence data on pure menstrual migraine and menstrually related migraine in the general population.

27. Tepper SJ, Zatochill M, Szeto M, et al.: Development of a simple menstrual migraine screening tool for obstetric and gynecology clinics: the menstrual migraine assessment tool. Headache 2008, 48:1419-1425.

28. Bekkelund SI, Alstadhaug KB, Salvesen R: Lack of seasonal variation in menstrually-related migraine. Cephalalgia 2008, 28:1277-1281.

29. Couturier EG, Bomhof MA, Neven AK, van Duijn NP: Menstrual migraine in a representative Dutch population sample: prevalence, disability and treatment. Cephalalgia 2003, 23:302-308.

30. Mattsson P: Hormonal factors in migraine: a population-based study of women aged 40 to 74 years. Headache 2003, 43:27-35.

31. Dzoljic E, Sipetic S, Vlajinac H, et al.: Prevalence of menstrually related migraine and nonmigraine primary headache in female students of Belgrade University. Headache 2002, 42:185-193.

32. Cupini LM, Matteis M, Troisi E, et al.: Sex-hormone-related events in migrainous females. A clinical comparative study between migraine with aura and migraine without aura. Cephalalgia 1995, 15:140-144.

33. Granella F, Sances G, Zanferrari C, et al.: Migraine without aura and reproductive life events: a clinical epidemiological study in 1300 women. Headache 1993, 33:385-389.

34. Beckham JC, Krug LM, Penzien DB, et al.: The relationship of ovarian steroids, headache activity and menstrual distress: a pilot study with female migraineurs. Headache 1992, 32:292-297.

35. MacGregor EA, Chia H, Vohrah RC, Wilkinson M: Migraine and menstruation: a pilot study. Cephalalgia 1990, 10:305-310. 